\title{
Chest Radiography in Coronavirus Disease 2019 (COVID-19): Correlation with Clinical Course
}

Joel C $\underline{\text { Zhou, }},{ }^{1} M D$, Terrence CH $\underline{\text { Hui }},{ }^{1} M B B S, F R C R$, Cher Heng Tan, ${ }^{1,2}{ }_{M B B S, F R C R}$, Hau Wei Khoo, ${ }^{1} M D, F R C R$, Barnaby E $\underline{\text { Young, }}{ }^{3,4,5}$ MB BChir, MSc, MRCP, David C Lye, ${ }^{2,3,4,5}$ MBBS, FAMS, FRCP, Yeong Shyan Lee, ${ }^{1} M B B C h, F R C R$, Gregory JL $\underline{\mathrm{Kaw}},{ }^{1}$ MBBS, FRCR, FAMS

\begin{abstract}
Coronavirus disease 2019 (COVID-19) is caused by the severe acute respiratory syndrome coronavirus 2 and was declared a global pandemic by the World Health Organization on 11 March 2020. A definitive diagnosis of COVID-19 is made after a positive result is obtained on reverse transcription-polymerase chain reaction assay. In Singapore, rigorous contact tracing was practised to contain the spread of the virus. Nasal swabs and chest radiographs (CXR) were also taken from individuals who were suspected to be infected by COVID-19 upon their arrival at a centralised screening centre. From our experience, about $40 \%$ of patients who tested positive for COVID-19 had initial CXR that appeared "normal". In this case series, we described the temporal evolution of COVID-19 in patients with an initial "normal" CXR. Since CXR has limited sensitivity and specificity in COVID-19, it is not suitable as a first-line diagnostic tool. However, when CXR changes become unequivocally abnormal, close monitoring is recommended to manage potentially severe COVID-19 pneumonia.
\end{abstract}

Ann Acad Med Singapore 2020;49:456-61

Key words: Diagnostic Radiology, Infectious Diseases, Pulmonary

\section{Introduction}

Coronavirus disease 2019 (COVID-19) is caused by the severe acute respiratory syndrome coronavirus 2 . Like other coronavirus infections, COVID-19 can cause fatal lower respiratory tract infection in the healthy population, although it appears to be less lethal than severe acute respiratory syndrome (SARS) and Middle East respiratory syndrome (MERS). ${ }^{1}$ On 11 March 2020, COVID-19 was declared a pandemic by the World Health Organization. ${ }^{2}$ Since the outbreak of this infectious disease, Singapore has reported $>55,000$ infected cases and 27 deaths. $^{3}$ Globally, there are $>18$ million cases of infection and $>730,000$ deaths. ${ }^{4}$

In Singapore, COVID-19 diagnosis is made through reverse transcription-polymerase chain reaction (RT-PCR) assays on nasopharyngeal swab specimens taken from individuals. ${ }^{5}$ On their arrival at the National Centre for Infectious Diseases (NCID), chest radiographs (CXR) were performed on them to detect pneumonia on imaging findings. After they were found to have a history of contact with patients who tested positive for COVID-19 and there was

${ }^{1}$ Department of Diagnostic Radiology, Tan Tock Seng Hospital, Singapore

${ }^{2}$ Lee Kong Chian School of Medicine, Nanyang Technological University, Singapore

${ }^{3}$ National Centre for Infectious Diseases, Singapore

${ }^{4}$ Department of Infectious Diseases, Tan Tock Seng Hospital, Singapore

${ }^{5}$ Yong Loo Lin School of Medicine, National University of Singapore, Singapore

Address for Correspondence: Dr Tan Cher Heng, Department of Diagnostic Radiology, Tan Tock Seng Hospital, 11 Jalan Tan Tock Seng, Singapore 308433.

Email: cher_heng_tan@ttsh.com.sg 
radiological evidence of pneumonia, they were classified as suspected COVID-19 cases and were admitted to await confirmatory results of RT-PCR assays.

In our centre, 1 patient who developed acute symptoms of fever and sore throat was discharged from the Emergency Department (ED) after the criteria for COVID-19 diagnosis were not met and findings of initial CXR were normal. Subsequently, the patient presented to another hospital and a definitive diagnosis of COVID-19 was made after CXR findings were positive for pneumonia. ${ }^{6}$ This near miss prompted us to review the first 40 COVID-19 cases who presented to the screening centre in NCID and after $40 \%$ of them had initial CXR that demonstrated either normal or subtle changes compared to community-acquired pneumonias, particularly those of bacterial origin. Duration from symptom onset to admission ranged between $0-9$ days.

In this study, we reviewed recent imaging findings of COVID-19 and discussed the roles and limitations of CXR to screen potential COVID-19 patients in relation to the temporal evolution of COVID-19. We also illustrated key learning points from CXR results of 5 COVID-19 patients in our case series.

\section{Case 1: Normal CXR During Hospitalisation}

A 31-year-old Chinese woman with a history of hypertension presented with dry cough and sore throat of 2 days' duration. Subsequently, she tested positive for COVID-19. At admission, her CXR findings were "normal". Serial CXR performed on days 6, 12 and 17 were also "normal" and showed no signs of infection.

She remained well throughout her hospitalisation and did not require supplemental oxygen therapy. At discharge, her symptoms had resolved completely and results of RT-PCR assays returned negative.

\section{Case 2: Normal CXR with Mild Disease}

A 48-year-old Chinese woman with no significant medical history presented with dry cough of 1 day's duration. Initial CXR (Fig. 1A) did not show any infection and she was discharged. Subsequently, she developed dyspnoea, mild fever of $37.5^{\circ} \mathrm{C}$ and left pleuritic chest pain. After she returned to NCID on day 10 of illness, CXR findings demonstrated new bilateral, multifocal middle to lower zone consolidation with no pleural effusion (Fig. 1B). She was isolated and subsequently tested positive for COVID-19 on RT-PCR assay.
Throughout her hospitalisation, normal oxygen saturation on room air was maintained and she did not require supplemental oxygen therapy. On day 35 , she was discharged after 2 consecutive RT-PCR assays returned negative. At 2 months, follow-up CXR showed complete resolution of airspace opacities (Fig. 1C).

\section{Case 3: Abnormal CXR with Disease Progression}

A 35-year-old man with no medical history presented with dry cough and fever. On day 3 of illness, he was admitted and tested positive for COVID-19. Initial CXR showed subtle ground-glass changes in the left lower zone (Fig. 2A). Although he remained stable throughout hospitalisation with improvement in symptoms, CXR on day 7 showed worsening ground-glass changes in the left lower zone (Fig. 2B) that developed into an area of consolidation on day 11 (Fig. 2C).

Throughout his hospital stay, he maintained oxygen saturation of $>94 \%$ on room air and did not require supplemental oxygen therapy. This was attributed to his young age and preserved lung functional reserve. On day 15, he was discharged after improvement in left lower zone consolidation was seen.

\section{Case 4: Normal CXR with Disease Progression and Clinical Deterioration}

A 56-year-old man with no medical history who developed fever and sore throat presented on day 3 of illness. Initial CXR was normal and he was discharged with symptomatic treatment. On day 8 , he was admitted after his symptoms persisted but CXR did not show any significant opacity (Fig. 3A). Two days later, however, CXR demonstrated new areas of consolidation in the right lower zone (Fig. 3B).

On day 13, he suffered an acute episode of desaturation that required supplemental oxygen therapy. Lowest oxygen saturation on room air was $89 \%$; subsequently, he required oxygen therapy with $35 \% \mathrm{FiO}_{2}$. CXR performed on the same day showed worsening bilateral lower zone consolidation (Fig. 3C). His clinical course was unremarkable and he was discharged on day 19. No follow-up CXR was performed.

\section{Case 5: Abnormal CXR with Disease Progression Requiring Intensive Care}

A 54-year-old man with no significant medical history presented with fever of 2 days' duration. At admission, CXR did not show lung opacity (Fig. 4A). On day 8, 

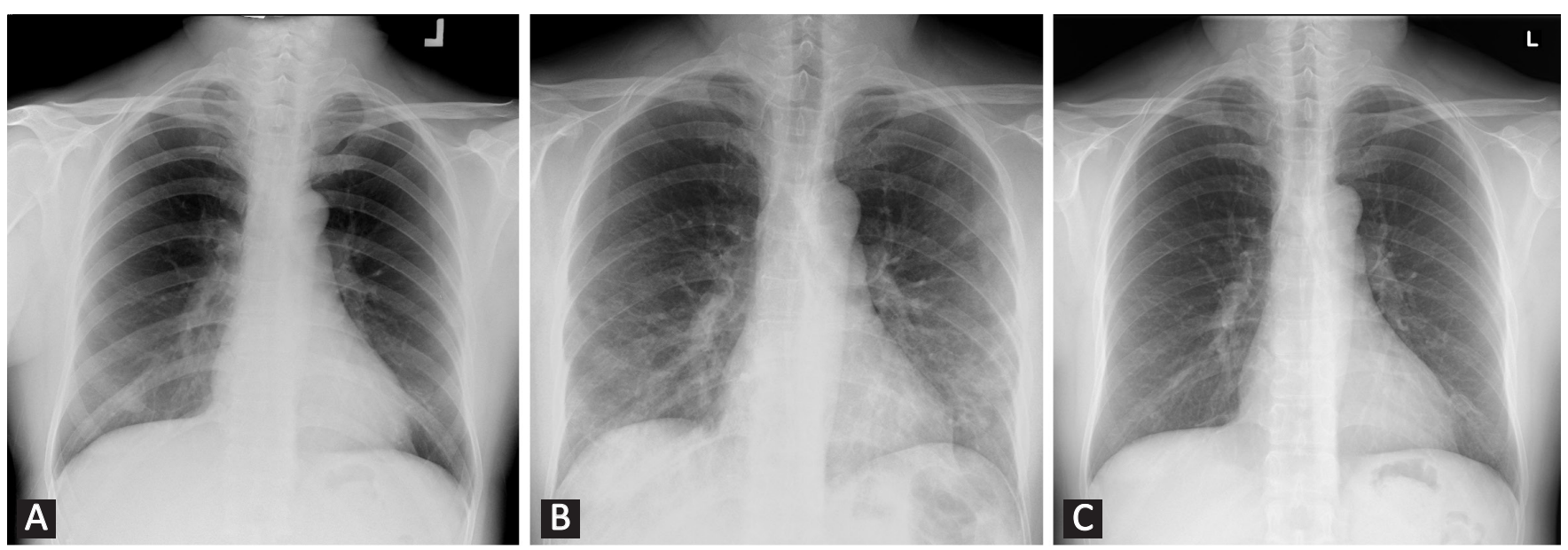

Fig. 1. CXR in patient with mild COVID-19. A: On day 1, CXR appeared normal. B: On day 10, development of bilateral middle to lower zone patchy consolidation was seen. C: At 2 months, lung consolidation had resolved completely. CXR: Chest radiograph; COVID-19: Coronavirus disease 2019
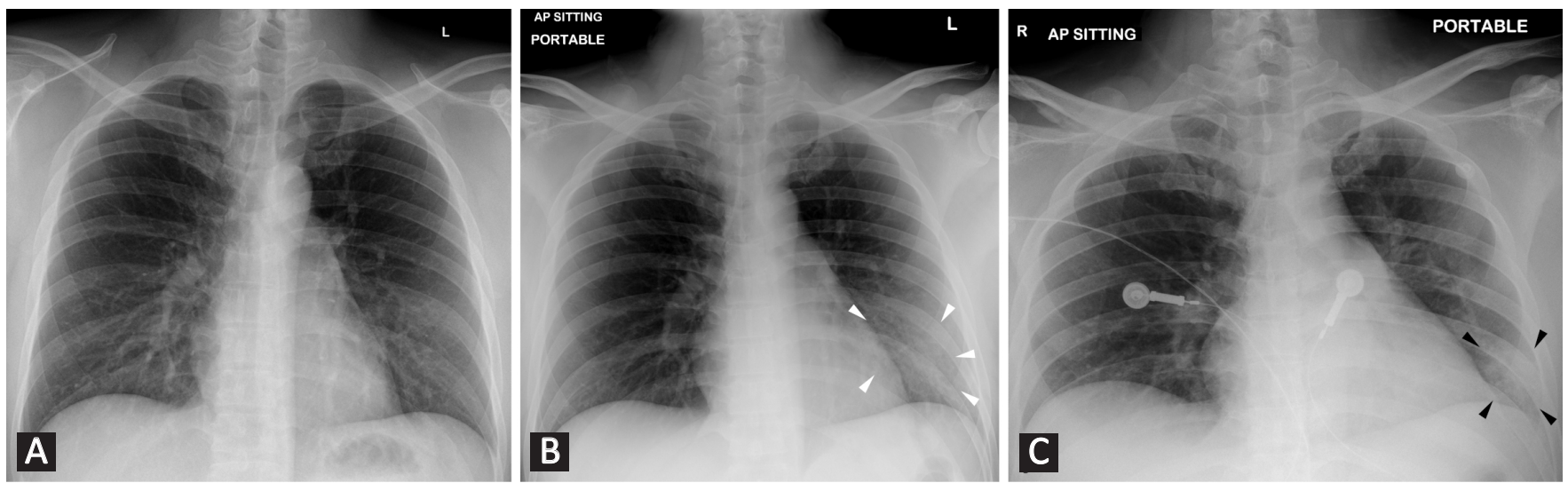

Fig. 2. CXR in patient with COVID-19 progression. A: On day 3, faint ground-glass changes in the left lower zone were seen. B: On day 7, ground-glass changes (white arrowheads) had worsened considerably. C: On day 11, an area of consolidation (black arrowheads) was seen. CXR: Chest radiograph; COVID-19: Coronavirus disease 2019
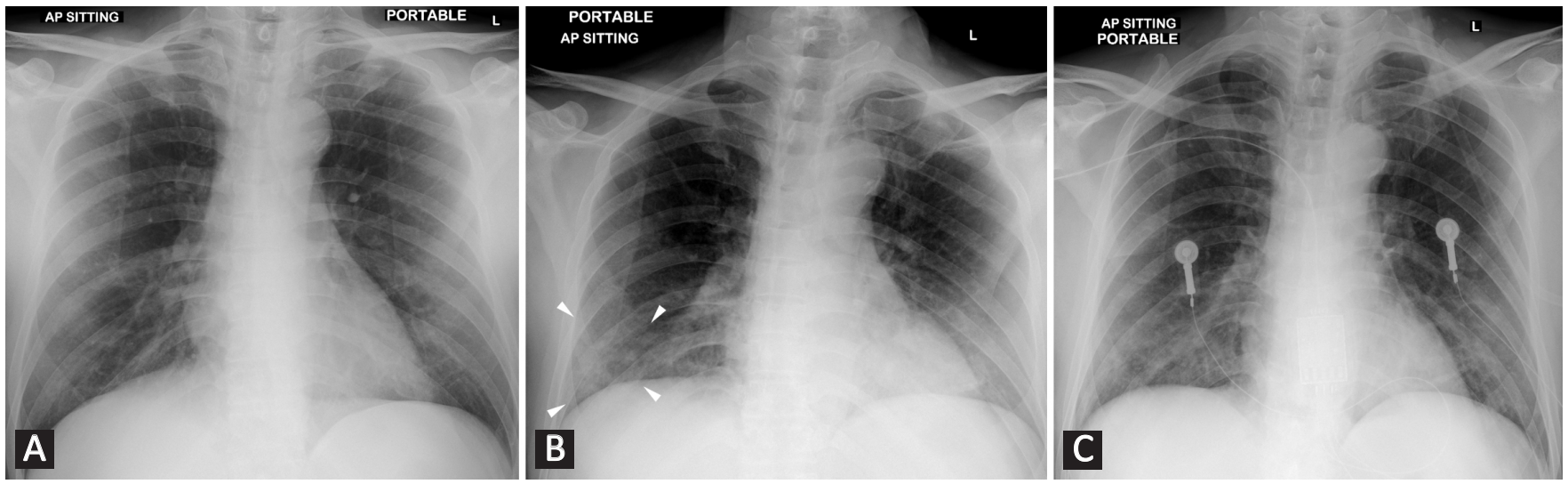

Fig. 3. CXR in COVID-19 patient with clinical deterioration. A: On day 8, CXR appeared normal. B: On day 10, development of new right lower zone consolidation (white arrowheads) was noted. C: On day 13, new bilateral lower zone consolidation was observed and patient desaturated during hospitalisation and required oxygen therapy. CXR: Chest radiograph; COVID-19: Coronavirus disease 2019 
he was transferred to Intensive Care Unit (ICU) after he developed increasing tachypnoea with desaturation (oxygen saturation on room air was $90 \%$ ) and oxygen therapy increased from $2 \mathrm{~L}$ to Venturi mask with $50 \%$ $\mathrm{FiO}_{2}$. Arterial blood gas showed type 1 respiratory failure and CXR showed new areas of consolidation in the left middle and bilateral lower zones (Fig. 4B).

In ICU, his condition deteriorated and he was placed on inotropic support and was intubated. On day 11, post-intubation CXR showed worsening bilateral lung consolidation (Fig. 4C) that improved on day 14. On day 32, he was discharged from ICU after 18 days. On day 46, CXR findings showed complete resolution of symptoms.

\section{Discussion}

Early symptoms in COVID-19 were described as non-specific and mild, and symptoms such as fever, dry cough and sore throat were commonly encountered. 5,7 With rigorous contact tracing and refinement in the definition of suspected COVID-19 cases, NCID has witnessed an exponential increase in the number of individuals who were sent to the centre for screening. To manage this high volume, a quick, affordable and reliable diagnostic kit is needed to accurately diagnose COVID-19 cases. $^{5}$

Although diagnostic kits differed among countries, RT-PCR assay is generally viewed as the method that yielded the most accurate results. ${ }^{8}$ While the lengthy wait for RT-PCR results has been reduced significantly since the outbreak of COVID-19, a time lag in practice is still seen. For example, the Fortitude Kit 2.0 developed by the Agency for Science, Technology and Research in Singapore - a diagnostic kit that was approved for use locally and internationally-required a waiting time of approximately 90 minutes before the results are available. ${ }^{9}$ Batch processing also contributed to a longer turnaround time.

Since the outbreak of the pandemic, CXR was used as a tool by NCID to determine whether suspected COVID-19 cases required hospitalisation. Initially, patients who were asymptomatic or with mild symptoms, no history of foreign travel and normal CXR findings were discharged with short-term follow-up. However, the limited sensitivity and specificity of CXR had led to several cases who subsequently tested positive for COVID-19 (such as cases 2 and 4). A multicentre study from China showed that $41 \%$ of patients with COVID-19 had normal CXR. ${ }^{10}$ Weinstock et al also found that approximately $60 \%$ of patients with COVID-19 who presented to ED had normal CXR. ${ }^{11}$

In the first 4 days of infection, findings on computed tomography (CT) scans typically showed groundglass opacities (GGO) in the subpleural regions of the lower lobes that involved 1 or both lungs. ${ }^{12}$ Similar CT findings in less severe disease were reported by $\mathrm{Ng}$ et $\mathrm{al}^{13}$ and Chung et al. ${ }^{14}$ In our experience, they typically appeared as subtle, hazy opacities on CXR. Between days 5-13, consolidation, multilobar distribution of GGO and crazy-paving pattern were seen. After the infection is controlled, resolution of the consolidation was seen. ${ }^{12}$ Despite the higher sensitivity of CT than CXR in imaging airspace opacities, COVID-19 is currently known to present with normal imaging findings, particularly during
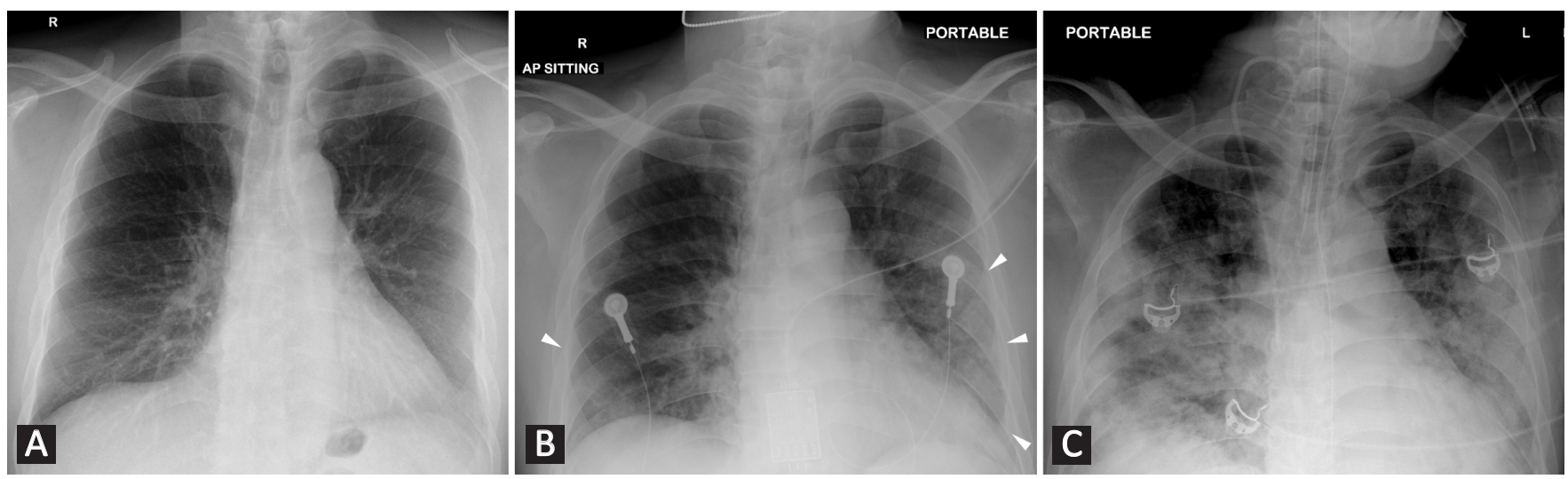

Fig. 4. CXR in COVD-19 patient admitted to the Intensive Care Unit. A: On day 2, no abnormalities were noted. B: On day 8, new areas of consolidation in the left middle and bilateral lower zones (white arrowheads) were observed. C: On day 11, progressive worsening of bilateral lung consolidation after intubation was seen. CXR: Chest radiograph; COVID-19: Coronavirus disease 2019 
the early phase of infection and in those with mild symptoms. ${ }^{15}$

Imaging features of COVID-19 are deemed to be non-specific and mimic atypical pneumonia that is caused by other pathogens such as SARS and MERS. Additionally, GGO and consolidation are commonly described in viral pneumonias caused by adenovirus, cytomegalovirus and herpes simplex virus. ${ }^{16}$

As the pandemic continues to evolve, the definition of local cases of suspected COVID-19 has, at the time of writing, been expanded to include individuals who showed signs and symptoms that are suggestive of community-acquired pneumonia or acute respiratory illness. While RT-PCR assay is used as the primary diagnostic tool, CXR is used to determine the extent of disease and to exclude other causes of respiratory symptoms. Since acute respiratory distress syndrome is reported as a potential complication in COVID-19 patients, ${ }^{17}$ CXR may be used to monitor disease progression during hospitalisation.

Our case series had demonstrated the low sensitivity of CXR to diagnose COVID-19 in the first few days of infection. Nevertheless, CXR was shown to be useful in imaging clinically significant lung impairment. When there was no disease progression after normal or mild findings were found on CXR, patients recovered after a relatively benign course of illness (cases 1 and 2). However, when disease progression in the form of bilateral consolidation with lower zone predominance that reached peak severity between days $10-12$ was present, ${ }^{18,19}$ patients deteriorated and required either supplemental oxygen therapy or mechanical ventilation (cases 3, 4 and 5).

In the early days of the COVID-19 outbreak in Hubei Province in Mainland China, physicians used chest $\mathrm{CT}$ to diagnose infection after they experienced an acute shortage of diagnostic kits and a spike in the number of suspected cases. ${ }^{20}$ In Singapore, however, 2 primary factors prevented the use of $\mathrm{CT}$ as a diagnostic tool in COVID-19. First, CT machines are a shared resource that is meant for use by all patients. When every suspected COVID-19 case is subjected to a CT scan, this will create a huge burden on the health system since there is a need to facilitate patient transfers, terminal cleaning and appropriate time gaps to avoid nosocomial transmission. This renders the use of $\mathrm{CT}$ as a screening tool impractical.

Second, findings on the specificity of CT in imaging lung changes are not known. Consequently, the use of CT as a screening tool may lead to false positive results and incur unnecessary expenditure and resource wastage. Additionally, in the first 2 days of disease, findings have shown that up to $56 \%$ of CT results were negative. ${ }^{21}$ Recently, the American College of Radiologists recommended that CT should not be used as a first-line screening and diagnostic tool, and should be reserved for use in symptomatic, hospitalised patients. ${ }^{22}$

For radiologists, the challenge in making a potential diagnosis of COVID-19 is the detection of airspace haziness. Since CXR is not sensitive in detecting GGO, findings of initial CXR may appear normal or show extremely subtle changes. Other factors such as patient characteristics and technical constraints may compromise the sensitivity of CXR. For example, in obese patients or patients with dense breast shadows, poor penetration of the peripheral lung fields can affect CXR findings. The presence of underlying lung pathologies such as bronchiectasis, pulmonary fibrosis or lung malignancy may also pose a diagnostic challenge. Conversely, asymmetric breast shadows may mimic disease. Heightened sensitivity in at-risk patients may lead radiologists to "over-call" CXR findings as positive. Consequently, optimal patient outcomes can only be achieved through close collaboration between clinicians and radiologists.

\section{Conclusion}

In the early stages of COVID-19, findings of CXR are mostly negative in approximately half of patients. In some patients, normal results or mild abnormalities are seen throughout the disease course and no specific therapy is required. However, when CXR findings are unequivocal for infection, rapid isolation and close monitoring should be instituted in at-risk patients even before the results of RT-PCR assay are known since CXR changes may portend clinical deterioration and need for oxygen therapy or mechanical ventilation. In the ongoing pandemic, clinicians and radiologists must be mindful of the role and limitations of CXR in the diagnosis and management of COVID-19 patients.

\section{REFERENCES}

1. Chen J. Pathogenicity and transmissibility of 2019-nCoV-a quick overview and comparison with other emerging viruses. Microbes Infect 2020;22:69-71.

2. World Health Organization. Coronavirus disease 2019 (COVID-19) Situation Report 51. Available at: https://www.who.int/docs/defaultsource/coronaviruse/situation-reports/20200311-sitrep-51-covid-19. pdf?sfvrsn=1ba62e57_10. Accessed on 1 April 2020. 
3. Ministry of Health, Singapore. Daily Report on COVID-19 as at 4 August 2020, 1200 hrs. Available at: https://www.moh.gov.sg/docs/ librariesprovider5/local-situation-report/20200804_daily_report_on covid-19 cabinet.pdf. Accessed on 5 August 2020.

4. World Health Organization. Coronavirus disease (COVID-19) Situation Report 197. Available at: https://www.who.int/docs/defaultsource/coronaviruse/situation-reports/20200804-covid-19-sitrep-197. pdf?sfvrsn=94f7a01d_2. Accessed on 5 August 2020 .

5. Wong JEL, Leo YS, Tan CC. COVID-19 in Singapore-current experience: critical global issues that require attention and action. JAMA 2020; doi:10.1001/jama.2020.2467.

6. Ministry of Health, Singapore. Confirmed cases of local transmission of novel coronavirus infection in Singapore. Available at: https:// www.moh.gov.sg/news-highlights/details/confirmed-cases-of-localtransmission-of-novel-coronavirus-infection-in-singapore. Accessed on 3 March 2020.

7. Huang C, Wang Y, Li X, Ren L, Zhao J, Hu Y, et al. Clinical features of patients infected with 2019 novel coronavirus in Wuhan, China. Lancet 2020;395:497-506.

8. Koh D, Cunningham AC. Counting coronavirus disease 2019 (COVID-19) cases: case definitions, screened populations and testing techniques matter. Ann Acad Med Singapore 2020;49:161-5

9. Agency for Science, Technology and Research, Singapore. Fighting COVID-19 with fortitude. Available at: https://www.a-star.edu.sg/Newsand-Events/a-star-news/news/covid-19/fighting-covid-19-with-fortitude. Accessed on 3 March 2020.

10. Guan WJ, Ni ZY, Hu Y, Liang WH, Ou CQ, He JX, et al. Clinical characteristics of coronavirus disease 2019 in China. New Engl J Med 2020;382:1708-20.

11. Weinstock MB, Echenique A, Russell JW, Miller JA, Cohen DJ, Waite S, et al. Chest X-ray findings in 636 ambulatory patients with COVID-19 presenting to an urgent care center: a normal chest X-ray is no guarantee. J Urgent Care Med 2020;14:13-18.

12. Pan F, Ye T, Sun P, Gui S, Liang B, Li L, et al. Time course of lung changes at chest CT during recovery from 2019 coronavirus diseae 2019 (COVID-19). Radiology 2020;295:715-21.
13. Ng MY, Lee EYP, Yang J, Yang F, Li X, Wang H, et al. Imaging profile of the COVID-19 infection: radiologic findings and literature review. Radiol Cardiothorac Imaging 2020;2:e200034.

14. Chung M, Bernheim A, Mei X, Zhang N, Huang M, Zeng X, et al. CT imaging features of 2019 novel coronavirus (2019-nCoV). Radiology 2020;295:202-7.

15. Inui S, Fujikawa A, Jitsu M, Kunishima N, Watanabe S, Suzuki Y, et al. Chest CT findings in cases from the cruise ship "Diamond Princess" with coronavirus disease 2019 (COVID-19). Radiol Cardiothorac Imaging 2020;2:e200110.

16. Koo HJ, Lim S, Choe J, Choi SH, Sung H, Do KH. Radiographic and CT features of viral pneumonia. Radiographics 2018;38:719-39.

17. Goh KJ, Choong MCM, Cheong EHT, Kalimuddin S, Duu Wen S, Phua GC, et al. Rapid progression to acute respiratory distress syndrome: review of current understanding of critical illness from COVID-19 infection. Ann Acad Med Singapore 2020;49:108-18.

18. Wong HYF, Lam HYS, Fong AHT, Leung ST, Chin TWY, Lo CSY, et al. Frequency and distribution of chest radiographic findings in patients positive for COVID-19. Radiology 2020;296:E72-8.

19. Hui TCH, Khoo HW, Young BE, Haja Mohideen SM, Lee YS, Lim CJ, et al. Clinical utility of chest radiography for severe COVID-19. Quant Imaging Med Surg 2020;10:1540-50.

20. Ai T, Yang Z, Hou H, Zhan C, Chen C, Lv W, et al. Correlation of chest CT and RT-PCR testing for coronavirus disease 2019 (COVID-19) in China: a report of 1014 cases. Radiology 2020; 296:E32-40

21. Bernheim A, Mei X, Huang M, Yang Y, Fayad ZA, Zhang N, et al. Chest CT findings in coronavirus disease-19 (COVID-19): relationship to duration of infection. Radiology 2020;295:200463.

22. American College of Radiology. Recommendations for the use of chest radiography and computed tomography (CT) for suspected COVID-19 infection. Available at: https://www.acr.org/Advocacy-andEconomics/ACR-Position-Statements/Recommendations-for-ChestRadiography-and-CT-for-Suspected-COVID19-Infection. Accessed on 4 April 2020. 